\title{
METODOLOGÍA EN OTORRINOLARINGOLOGÍA. LECTURA CRÍTICA DE LA LITERATURA: IMPORTANCIA Y APLICABILIDAD
}

\section{Methodology in Otorhinolaryngology. Critical Reading of Literature: Importance and Applicability}

\author{
Luis Miguel PALOMAR-RODRÍGUEZ \\ Complejo Asistencial de Zamora. Servicio de Medicina Interna. Zamora. España. \\ Correspondencia: Impalomar@telefonica.net
}

Fecha de recepción: 28 de junio de 2017

Fecha de aceptación: 14 de julio de 2017

Fecha de publicación: 15 de julio de 2017

Fecha de publicación del fascículo: 1 de diciembre de 2018

Conflicto de intereses: Los autores declaran no tener conflictos de intereses

Imágenes: Los autores declaran haber obtenido las imágenes con el permiso de los pacientes Política de derechos y autoarchivo: se permite el autoarchivo de la versión post-print (SHERPA/RoMEO)

Licencia CC BY-NC-ND. Licencia Creative Commons Atribución-NoComercial-SinDerivar 4.0 Internacional Universidad de Salamanca. Su comercialización está sujeta al permiso del editor

RESUMEN

PALABRAS CLAVE

SUMMARY

KEYWORDS
Introducción y objetivo: La lectura crítica de la literatura es una habilidad deseable en todo médico, que se debería adquirir en el contexto de la formación de todos los MIR, pero también en todos los ya pasaron por este proceso y no la adquirieron. Objetivo: Desarrollar el concepto de lectura crítica. Conclusiones: Aunque a priori puede generar rechazo en muchos por considerarlo inalcanzable, realmente es relativamente sencillo, no requiere una formación profunda en metodología, epidemiología y estadística, y existen hoy en día muchas herramientas de ayuda para aplicarla con facilidad. Está demostrado que aporta una visión crítica al médico, lo que se considera fundamental, sirve para mantenerse actualizado, es un estímulo de autosuperación y genera satisfacción en el profesional.

metodología; lectura crítica; CASPE

Introduction and objective: The critical appraisal of the literature is a desirable skill in every doctor, who should be acquired in the context of the formation of all the MIR, but also in all either they happened for this process and did not acquire it. Objective: Develop the concept of critical reading. Conclusions: Although beforehand it can generate rejection in many for considering it to be unattainable, really it is relatively simple, it does not need a deep formation in methodology, epidemiology and statistics, and many help hardware exists nowadays to apply it with facility. It is demonstrated that it brings a critical vision to the doctor, what is considered to be fundamental, serves to stay updated, it is an autoovercoming stimulus and it generates satisfaction in the professional.

methodology; critical reading of literature; CASPE 


\section{INTRODUCCIÓN}

Recientemente leí en un artículo publicado en el País, titulado "Alerta, pregunta incómoda: ¿está fracasando la medicina?» [1], algo que me llamó la atención y fue que hablaban del concepto de medicina basada en la evidencia (MBE), a raíz de un artículo publicado en BMJ por Trisha Greenhalgh, la autora del célebre libro: How to read a paper. The basics of evidence based medicine [2]. Dicho artículo se titulaba: Evidence based medicine: a movement in crisis? En él argüía que, aunque la medicina basada en la evidencia ha traído muchos beneficios, también ha generado consecuencias negativas, y por ello, se requiere un renacimiento $y$ un reenfoque que combine adecuadamente la relación entre un médico experimentado y un paciente para ofrecerle un tratamiento adecuado ( $y$ un pronóstico, un riesgo, una prueba diagnóstica...). La lectura crítica de la literatura científica en medicina está íntimamente ligada a la medicina basada en la evidencia, dado que aquella es un requisito fundamental para aplicar la mejor evidencia disponible a nuestros pacientes, difícilmente sabremos si un artículo es una buena evidencia sino tenemos la habilidad de realizar una lectura crítica.

\section{¿QUÉ ES LA LECTURA CRÍTICA?}

Lo primero que hay que valorar es la validez en un artículo, es decir, si las conclusiones del estudio se acercan a la verdad, o lo que es lo mismo, si hay ausencia de sesgos o errores sistemáticos. Si existen sesgos o errores sistemáticos, las conclusiones pueden estar más o menos alejadas de la verdad, y lo peor, no sabemos cuánto están alejadas. Para conocer e interpretar la validez de un estudio se requieren unos mínimos conocimientos o habilidades, además existen guías o cheklist que nos ayudan en este sentido, como la organización CASPe [3], The User's Guides to the Medical Literature [4].

Un médico puede leer literatura médica por varios motivos (Tabla 1) [6]. De ellos los que más nos interesan para la lectura crítica son los del 5 al 9 incluidos.

La interpretación de la evidencia se ha considerado, tradicionalmente, un asunto trivial asociado al sentido común clínico. Sin embargo, dos circunstancias hacen que ya no pueda ser visto de ese modo: por una parte, la aparición de modernos métodos de investigación (vinculados al extraordinario progreso de la epidemiologia clínica) que atañen tanto al diseño de estudios clásicos como al de nuevos estudios de síntesis, y también a la producción de sumarios de evidencia y guías de práctica clínica. Por otra, el desarrollo de nuevos modos de presentar la evidencia, con aparición de nuevas convenciones de escritura y de nuevos tipos de documentos (estudios, sinopsis, síntesis, sumarios). Ambas circunstancias hacen que la lectura crítica deba ser específicamente entrenada.

\section{Tabla 1. Diez razones para leer revistas clínicas}

\section{Para impresionar a otros}

2. Para estar al día de las novedades profesiones

3. Para comprender la patología

4. Para saber cómo maneja un problema determinado un clínico experimentado

5. Para saber cómo funciona una nueva prueba diagnóstica en un paciente

6. Para aprender los datos clínicos y la evolución de una enfermedad

7. Paraconocer la etiología o la causa de una patología

8. Para distinguir la utilidad de la inutilidad e incluso los efectos adversos de un tratamiento

9. Para aclarar conceptos sobre la necesidad de utilizar cuidados sanitarios, su calidad y coste efectividad

10. Para despertar el interés en las cartas al editor

Pero, en simetría con el comentario platónico, tampoco el lector elige sensu stricto al texto: hay todo un camino previo en el que el texto ha de sortear el entramado editorial y aun así su llegada al lector está a merced de las distintas estrategias usadas para difusión de la evidencia por parte de los diferentes agentes (editores, industria, sistemas de salud, etc.) lo que hace que esa elección tampoco sea realmente activa. En cuanto al camino del texto, no hay pruebas de que el sistema peer review garantice la calidad de lo publicado. Adicionalmente, hay evidencias crecientes que apuntan a que la elección de algunos diseños concretos, algunos tipos de financiación de los estudios y otros múltiples factores externos son capaces de afectar sustancialmente a los resultados de la investigación y, por supuesto, de condicionar su difusión.

Así pues, porque la investigación clínica es ahora diferente y porque el contexto de acceso y difusión a la evidencia cambia vertiginosamente, ya no basta el sentido común clínico para leer la evidencia publicada, y la lectura crítica de la evidencia emerge como una habilidad básica para la clínica del siglo XXI [5]. 
La segunda cuestión para valorar en un artículo son los resultados, y especialmente no solo la significación estadística $(p<0.05, p$. ej.), sino la magnitud de dichos resultados y su relevancia clínica.

Tras considerar la validez de un artículo, hay que pasar a considerar la validez externa o generalización, o en el caso de un paciente concreto, considerar la aplicabilidad para ese paciente. Para ello, hay que valorar múltiples factores, como la similitud de mi o mis pacientes a los del estudio, pronóstico y gravedad, posibilidades del tratamiento, prueba diagnóstica en mi entorno, efectos adversos, riesgo beneficio y otros factores más. De todo ello podremos encontrar más detalles en las guías referidas previamente y en libros como el que inició el Dr. Sackett y su grupo: EvidenceBased Medicine: How to practice and teach it, que ya va por su cuarta edición [7]. Reencontrándonos de nuevo con la MBE, en este punto tenemos que considerar las preferencias, los valores y creencias de los pacientes para aplicar un tratamiento o practicar una prueba diagnóstica.

¿QUÉ IMPORTANCIA TIENE PARA LA PRÁCTICA MÉDICA?

Responder a esta cuestión podría ser tan fácil y corto como decir «toda». Si bien es cierto que para aplicar las mejores evidencias en tratamientos, pruebas diagnósticas, pronóstico, etiología y riesgo entre otras, hoy en día podemos mirar en bases de datos actualizadas, referenciadas y con grados de evidencia y recomendaciones, como UpToDate, o en revistas 0 bases de datos procesadas o evaluadas por expertos en MBE (ACP Journal Club, Evidence Based Medicine, Essential Evidence Plus, Clinical Evidence), debemos adiestrarnos en la lectura crítica por múltiples motivos:

a. Muchas cuestiones que se nos plantean en la práctica clínica no están reflejadas en las fuentes comentadas previamente.

b. Nos da autonomía para tomar decisiones.

c. Nos permite mejorar en nuestra práctica clínica.

d. Nos ayuda a mantenernos actualizados y críticos.

e. Nos plantea retos de autosuperación.
¿QUÉ IMPORTANCIA TIENE PARA MEJORAR LA DOCUMENTACIÓN?

Los médicos, en la práctica clínica reciben información de dos fuentes principalmente: los pacientes y la documentación. De los primeros obtenemos datos desde la anamnesis, la exploración y diferentes pruebas, de la segunda. obtenemos información científica. Hoy en día, disponemos de bases de datos (en el amplio sentido de la palabra), bien en papel y sobre todo en formato electrónico, ya sean libros, revistas secundarias, revistas con artículos originales, publicaciones de la Colaboración Cochrane, Pubmed, UpToDate, metabuscadores como TRIP database, entre otras. De todas ellas tenemos que tener una visión crítica, es decir, valorar la validez y la aplicabilidad externa. Precisamente, la visión de la lectura crítica de la literatura y la MBE ha constituido el motor para que se elaboren consensos para la publicación de diferentes tipos de estudios clínicos: QUORUM y luego PRISMA para revisiones sistemáticas, CONSORT para ensayos clínicos, STROBE para estudios observacionales, STARD para pruebas diagnósticas y GRADE para graduar la calidad de la evidencia y fuerza de las recomendaciones [8-12].

A todos estos consensos se han ido sumando las editoras de las principales publicaciones en mayor o menor medida, permitiendo una mayor homogeneización y comparabilidad de la literatura médica. El adquirir estas habilidades también nos permite mejorar la calidad de nuestra propia documentación para documentar un artículo propio, por razones obvias.

¿CÓMO PODEMOS APLICARLO EN NUESTRA LECTURA INDIVIDUAL?

Evidentemente adquiriendo estas habilidades para la lectura crítica. Muchos dirían que los esfuerzos para conseguir estas habilidades los hacen inalcanzables, o que los conocimientos epidemiológicos y estadísticos que se requieren harían desfallecer a muchos: nada más alejado de la realidad. Es cierto que se requiere algún esfuerzo, algunos conocimientos epidemiológicos de diseño de estudio y algo de estadística, pero realmente es mínimo. Además, las guías disponibles para la lectura crítica facilitan mucho el proceso. El aprendizaje tutorizado en seminarios y cursos también se considera de gran ayuda. 
Simplificando el proceso, la lectura crítica de la literatura se basa en tres preguntas sencillas:

- ¿Son válidos los resultados del estudio?

- ¿Cuáles fueron los resultados?

- ¿Pueden estos resultados ser útiles en el cuidado de mis pacientes?

Estos tres bloques están reflejados en las guías publicadas inicialmente en 1981 en la revista Canadian Medical Association Journal y actualizadas en una nueva serie publicada desde 1993 en JAMA y que se recoge en The User's Guides to the Medical Literature [4].

Para evaluar los resultados y como aplicarlos, nos basamos en la asunción de que la fuerza o consistencia de un estudio está relacionada con el diseño del estudio y este varía según el tipo de interrogante que planteemos (diagnóstico, prevención y tratamiento, curso y pronóstico y efectos perjudiciales de las enfermedades).

La primera parte de la evaluación se puede lograr aplicando unos criterios sencillos, distintos según el tipo de estudio de que se trate, que permiten de una forma rápida (incluso leyendo únicamente el resumen) seleccionar de los documentos encontrados los más válidos, desechando los que no cumplen estos criterios y reduciendo por tanto el número de artículos que necesitan una lectura más profunda.

Muchos artículos se presentan con el formato IMRAD ○ IMRyD (en español): Introducción, Métodos, Resultados y Discusión. Si tú decides que el artículo te interesa, por el título y el resumen, deberías mirar en métodos la respuesta a la primera pregunta y después los resultados. La importancia, la magnitud y la precisión de estos resultados, es fácil evaluarlos, aunque a veces los autores nos lo ponen un poco más difícil. La respuesta a la última pregunta es una valoración del parecido o similitud de los pacientes del estudio a los míos, y de otros aspectos como recursos, accesibilidad y coste beneficio entre otros.

\section{BIBLIOGRAFÍA}

1. Suleng K. Alerta, pregunta incómoda: ¿está fracasando la medicina? El país [Internet] 4 sep 2016. Disponible en: http://elpais.com/elpais/2016/08/31/buenavida/ 1472648769_377736.html. [Último acceso: 13 de julio de 2017].
2. How to Read a Paper: The Basics of Evidence-Based Medicine, 5th Edition Trisha Greenhalgh. March 2014, BMJ Books

3. CASPe: Programa de Habilidades en Lectura Crítica Español. Alicante (España). Disponible en: http://www.redcaspe.org/. [Último acceso: 13 de julio de 2017].

4. User's Guides to the Medical Literature. A manual for evidence-based clinical practice, 3rd edition. McGraw Hill Education. JAMA evidence.

5. Cabello López, JC. Lectura crítica de la evidencia clínica. Elsevier, 2015.

6. How to read clinical journals: I. why to read them and how to start reading them critically. an Med Assoc J. 1981;124(5):555-8.

7. Straus S, Richardson WS, Glasziou P, Haynes RB. Evidence-based medicine: How to practice and teach EBM (4rd Ed.). Churchill Livingstone. Edinburgh, 2011.

8. Liberati A, Altman DG, Tetzlaff J, Mulrow C, Gøtzsche PC, loannidis JP, et al. The PRISMA statement for reporting systematic reviews and meta-analyses of studies that evaluate health care interventions: explanation and elaboration. PLoS Med. 2009;6(7):e1000100. doi: 10.1371/journal.pmed. 1000100.

9. Moher D, Schulz KF, Altman DG. The CONSORT statement: revised recommendations for improving the quality of reports of parallel-group randomised trials. Lancet. 2001; 357:1191-4.

10. Von Elm E, Altman DG, Egger M, Pocock SJ, Gøtzsche PC, Vandenbroucke JP; STROBE Initiative. The Strengthening the Reporting of Observational Studies in Epidemiology (STROBE) statement: guidelines for reporting observational studies. Lancet. 2007;370(9596):1453.

11. Bossuyt PM, Reitsma JB, Bruns DE, Gatsonis CA, Glasziou PP, IrwigLM, et al. Towards complete and accurate reporting of studies of diagnostic accuracy: the STARD Initiative. BMJ. 2003;326(7379):41-4.

12. Atkins $D$, Best $D$, Briss $P A$, Eccles $M$, FalckYtter Y, Flottorp S, et al; GRADE Working Group. Grading quality of evidence and strength of recommendations. BMJ 2004; 328:1490-2004. 